\title{
Allicin Protects against Lipopolysaccharide-Induced Acute Lung Injury by Up-Regulation of Claudin-4
}

\author{
Yue-liang Zheng, Wen-wei Cai, Guang-zhao Yan, Yuan-zhan Xu and Mei-qi \\ Zhang* \\ Department of Emergency, Zhejiang Provincial People's Hospital, Hangzhou 310014, China
}

*For correspondence: Email: meiqizhang1208@153.com; Tel: +86-0571-85893631

Received: 8 January 2014

Revised accepted: 31 May 2014

\begin{abstract}
Purpose: To investigate the effect of allicin, an active component of garlic, on lipopolysaccharide (LPS)induced acute lung injury.

Methods: Wistar rats were subjected to LPS intravenous injection with or without allicin treatment to induce acute lung injury (ALI) model. Also, A549 cells were stimulated with LPS in the presence and absence of allicin. HE staining was used to detect pathological changes in lung tissues. Enzyme-linked immunosorbent assay (ELISA) was performed to measure cytokine content. Cell viability was measured by CCK-8 and EdU incorporation assay. Genes expression was determined by real time polymerase chain reaction (PCR) and Western blot. Flow cytometry was applied to measure cell apoptosis.

Results: In vivo data showed that pulmonary edema, inflammatory cytokines expression and pathological changes were significantly attenuated in LPS-induced ALI after treatment with allicin $(p<$ $0.05)$ while in vitro results indicate that allicin administration significantly improved the $A 549$ cell viability in a dose-dependent manner as measured by CCK-8 and EdU incorporation assay. Besides, flow cytometry analysis showed that cell apoptosis rate was significantly reduced in a concentrationdependent manner after allicin injection (30.3 vs. $11.8 \%, p<0.05)$. At the molecular level, allicin treatment dose-dependently up-regulated claudin-4 expression both in vivo and in vitro $(p<0.05)$.

Conclusion: The findings indicate that allicin can protect against LPS-induced ALI in vivo and in vitro probably by up-regulation of claudin-4 expression.
\end{abstract}

Keywords: Allicin, Acute lung injury, Lipopolysaccharide, Claudin-4, Up-regulation, Pulmonary edema, Inflammatory cytukines

Tropical Journal of Pharmaceutical Research is indexed by Science Citation Index (SciSearch), Scopus, International Pharmaceutical Abstract, Chemical Abstracts, Embase, Index Copernicus, EBSCO, African Index Medicus, JournalSeek, Journal Citation Reports/Science Edition, Directory of Open Access Journals (DOAJ), African Journal Online, Bioline International, Open-J-Gate and Pharmacy Abstracts

\section{INTRODUCTION}

Acute lung injury (ALI) is characterized by acute respiratory distress syndrome, which may be induced by several situations such as trauma, sepsis and pneumonia [1]. It is a devastating clinical problem, with mortality rates as high as $60 \%$. Despite significant advances in antimicrobial supportive care and therapy to improve the survival of ALI patients, the mortality rate among ALI patients still remains high [2].
Therefore, it is imperative to find new and effective therapeutic drugs for the treatment of ALI.

Increasing evidences have shown that many proinflammatory cytokines play important roles in initiating and amplifying the lung injury [3]. Clinical data have also indicated that the proinflammatory cytokines elevation in the serum of ALI patients has been associated with increased mortality rate [4]. Many natural 
products possess immunomodulatory activities, which have long been sought for treating human diseases. Garlic (Allium sativum) is one of the most ancient vegetables and possesses evident pharmacological properties, such as antimicrobial, antioxidant and immunoregulatory activities [5]. Allicin is a major component and thiosulphinate compound responsible for the biological activity of garlic. Kim et al showed that combination of allicin with antifungal drugs could be effective in treating fungal infections with minimal side-effects [6]. However, the protective effect of allicin in LPS-induced acute lung injury has never been investigated. The present study is aimed at evaluating the therapeutic effect of allicin in LPS-induced acute lung injury in vivo and in vitro.

\section{EXPERIMENTAL}

\section{Model establishment}

Forty male Wistar rats were randomly divided into 3 groups $(n=10)$, control group, ALI group and allicin group. According to the previous report, ALI was induced by LPS (Sigma, St Louis, MO, USA) via intratracheal injection [7]. In brief, rats were anesthetized with pentobarbital sodium, followed by LPS intratracheal injection at a dose of $5 \mathrm{mg} / \mathrm{kg}$. Then, the rats were placed in a vertical position and rotated for 1 minute to distribute the instillation in the lungs. Ten minutes after LPS injection, allicin was given by intravenous injection in the dorsal penile vein at a dose of $30 \mu \mathrm{g} / \mathrm{kg}$. All animal experiments were performed in accordance with the approval received from the Institutional Animal Ethics Committee following the guidelines of the Committee for the Purpose of Control and Supervision of Experiments on Animals (CPCSEA), Government of China (NO.:IACUC2012-018) and the International Guidelines for Handling of Laboratory Animals [8].

\section{Histologic examination}

Rats were anesthetized at $24 \mathrm{~h}$ after injury and killed transcardially with saline, followed by treatment with $4 \%$ paraformaldehyde. Lungs were immediately removed and post-fixed in $4 \%$ paraformaldehyde for $24 \mathrm{~h}$. Paraffin-embedded sections with thickness of $5 \mathrm{~mm}$ were stained with hematoxylin and eosin (HE) for visualization under a light microscope (Leica Microsystems, Wetzlar, Germany) at 200 x magnification.

\section{Enzyme-linked immunosorbent assay}

Claudin-4, TNF- $\alpha$, IL- 6 and TGF- $\beta 1$ levels in lung tissue samples were measured using enzyme- linked immunosorbent assay (ELISA) according to its manufacturer's instructions (R\&D Systems, Minneapolis, MN, USA). Absorbance was measured at $450 \mathrm{~nm}$ by microplate assay.

\section{Lung wet/dry weight ratio}

The severity of pulmonary edema was assessed by the wet to dry weight ratio (W/D ratio). The left lower lungs were weighed and then dehydrated at $60{ }^{\circ} \mathrm{C}$ for $72 \mathrm{~h}$ in an oven.

\section{Cell culture and treatments}

Human type II-like alveolar epithelial cells A549 were cultured in RPMI1640 supplemented with $10 \%$ fetal bovine serum, $2 \mathrm{mmol} / \mathrm{L}$ glutamine, penicillin (100 U/mL) and streptomycin (100 $\mathrm{mg} / \mathrm{mL}$ ), and maintained at $37{ }^{\circ} \mathrm{C}$ and $5 \% \mathrm{CO}_{2}$ in a humid environment. Then, cells were treated with LPS (10 $\mu \mathrm{g} / \mathrm{mL})$ and different concentrations of allicin $(0 \mu \mathrm{g} / \mathrm{ml}, 25 \mu \mathrm{g} / \mathrm{ml}, 50$ $\mu \mathrm{g} / \mathrm{ml}$ and $100 \mu \mathrm{g} / \mathrm{ml}$ ). Twenty four hours later, cells were collected for the following experiments.

\section{Cell viability assay}

Cell viability was evaluated by the CCK-8 assay. In brief, cell density of $3 \times 10^{3}$ cells/well was seeded into 96-well plates and left to adhere overnight. The cells were then incubated with or without allicin. Then $100 \mu \mathrm{L}$ CCK-8 was added and incubated in the dark at $37{ }^{\circ} \mathrm{C}$ for $3 \mathrm{~h}$. The absorbance was determined on MRX II microplate reader (Dynex, Chantilly, VA, USA) at $450 \mathrm{~nm}$.

\section{EdU incorporation assay}

A549 cells were exposed to EdU (5-ethynyl-2'deoxyuridine) (Invitrogen, Carlsbad, CA, USA) for $2 \mathrm{~h}$ at $37{ }^{\circ} \mathrm{C}$. The cells were fixed with $4 \%$ formaldehyde for $15 \mathrm{~min}$ and treated with $0.5 \%$ Triton X-100 for $20 \mathrm{~min}$ at room temperature. After washing three times with phosphate buffered saline, the cells of each well were reacted with $100 \mu \mathrm{L}$ of $1 \mathrm{X}$ Apollo reaction cocktail for $30 \mathrm{~min}$. Subsequently, the DNA contents of the cells in each well were stained with $100 \mu \mathrm{L}$ of Hoechst $33342(5 \mu \mathrm{g} / \mathrm{mL})$ for 30 $\min$ and visualized under a fluorescent microscope (Leica Microsystems, Wetzlar, Germany).

\section{Real time PCR}

Total RNA was extracted with a TRIzol kit (Invitrogen Carlsbad, CA, USA) and converted to cDNA with a first strand cDNA synthesis kit 
(Fermentas, Burlington, Canada). Real time PCR was performed using SYBR Green SuperMix-UDG (Invitrogen Carlsbad, CA, USA). The primer sequences used for $\mathrm{PCR}$ are as follows: claudin-4 (forward 5'-CCTTTCCC ATACGGTCTTGCT-3', reverse 5'-CCCGTACC TTCCACAGACTG-3'), GAPDH (forward 5'CAGTGCCAGCCTCGTCTCATA-3', reverse 5'TGCCGTGGGTAGAGTCATA-3'). Amplification was performed at $50{ }^{\circ} \mathrm{C}$ for $2 \mathrm{~min}$, at $95^{\circ} \mathrm{C}$ for 2 min, followed by 40 cycles of denaturing at $95^{\circ} \mathrm{C}$ for $15 \mathrm{~s}$ and annealing at $60{ }^{\circ} \mathrm{C}$ for $30 \mathrm{~s}$. All reactions were performed in triplicate. Glyceraldehyde-3-phosphate dehydro-genase (GAPDH) was used as a reference gene.

\section{Western blot analysis}

Total protein $(20 \mu \mathrm{g})$ was separated from each sample by electrophoresis on a $4 \%-20 \%$ SDSpolyacrylamide gel and electroblotted onto polyvinylidene difluoride membranes. Membranes were blocked in a blocking solution, incubated overnight with primary antibodies including anti-claudin-4 and anti-GAPDH (Cell Signaling, Beverly, MA, USA). Anti-rabbit or antimouse secondary antibody conjugated with horseradish peroxidase was also used (Pierce Chromatography Cartridges, USA). Immunoreactive bands were detected by enhanced chemiluminescence (ECL) kit for Western blotting detection by using a ChemiGenius bioimaging system (SYNGENE, USA). Band densities of each protein were determined by Image-Pro Plus 6.0 (Media Cybernetics, Inc, Bethesda, MD, USA) with GAPDH used as control.

\section{Flow cytometry analysis}

Cells were exposed to different concentrations of allicin for $24 \mathrm{~h}$. The cells were washed with PBS, detached with trypsin and harvested. The cells were resuspended in $1 \mathrm{ml}$ Hoechst 33258 for 5 min then washed three times with PBS. Apoptotic cells were detected with annexin V-FITC/PI according to the protocol of Annexin V-FITC cell Apoptosis Detection Kit (BD, USA).

\section{Statistical analysis}

For statistical analysis, all the data were presented as means \pm SD using SPSS 13.0 program. Comparison between groups was made using ANOVA and statistically significant difference was defined as $p<0.05$.

\section{RESULTS}

\section{Allicin attenuated $A L I$ in rats stimulated by LPS in vivo}

To assess the pathological changes, HE staining was performed and results revealed evidence of notable inflammatory cells infiltration, interstitial edema, interalveolar septal thickening as well as intra-alveolar and interstitial hemorrhage. However, after allicin treatments, the pathological changes in the lung tissues were remarkably relieved (Fig 1A). Then, lung wet/dry weight ratio (W/D) was employed to evaluate the pulmonary edema. The rats that received LPS injection showed higher W/D than that in controls. However, W/D was significantly decreased after allicin administration (Fig 1B). Moreover, ELISA showed that the expression of inflammatory cytokines IL-6 and TNF- $\alpha$ in BALF in the rats with LPS injection were markedly higher than the rats in control group. However, IL-6 and TNF- $\alpha$ in BALF in the rats with allicin injections were significantly decreased (Fig $1 \mathrm{C}$ and D). Meanwhile, the anti-inflammatory mediator TGF$\beta 1$ was decreased in BALF in the rats with LPS injection, which was reversed by allicin treatment (Fig 1E). Together, these results demonstrate that allicin treatment alleviated LPS-induced ALI in vivo.

\section{Effect of allicin administration on A549 cell viability and apoptosis}

The lung epithelial cell line A549 was used in our study to investigate the role of allicin on A549 cell viability and apoptosis. CCK-8 assay revealed that the cell viabilities were significantly reduced after LPS treatment. Meanwhile, allicin administration significantly improved the A549 cell viabilities in a dose dependent manner (Fig 2A). EdU assay also showed similar results (Fig $2 \mathrm{~B}$ and $\mathrm{C}$ ). Flow cytometry was used to determine the effect of allicin on cell apoptosis. Results showed that allicin treatment significantly reduced A549 cells apoptosis rate compared to the controls (Fig 2D). These findings suggest that allicin could protect against ALI in A549 cells stimulated by LPS in vitro.

\section{Effect of allicin on claudin-4 expression in vivo and in vitro}

ELISA showed that expression of claudin-4 in BALF in the rats with LPS injection were markedly lower than the rats in control group. However, claudin-4 in BALF in the rats with allicin injections were significantly increased (Fig $3 A$ ). Besides, the mRNA and protein expressions 
of claudin-4 were significantly reduced in rat lung tissues with LPS injection. Nevertheless, allicin administration significantly increased the claudin-
4 expression as shown by real time PCR and Western blot (Fig 3B-D).

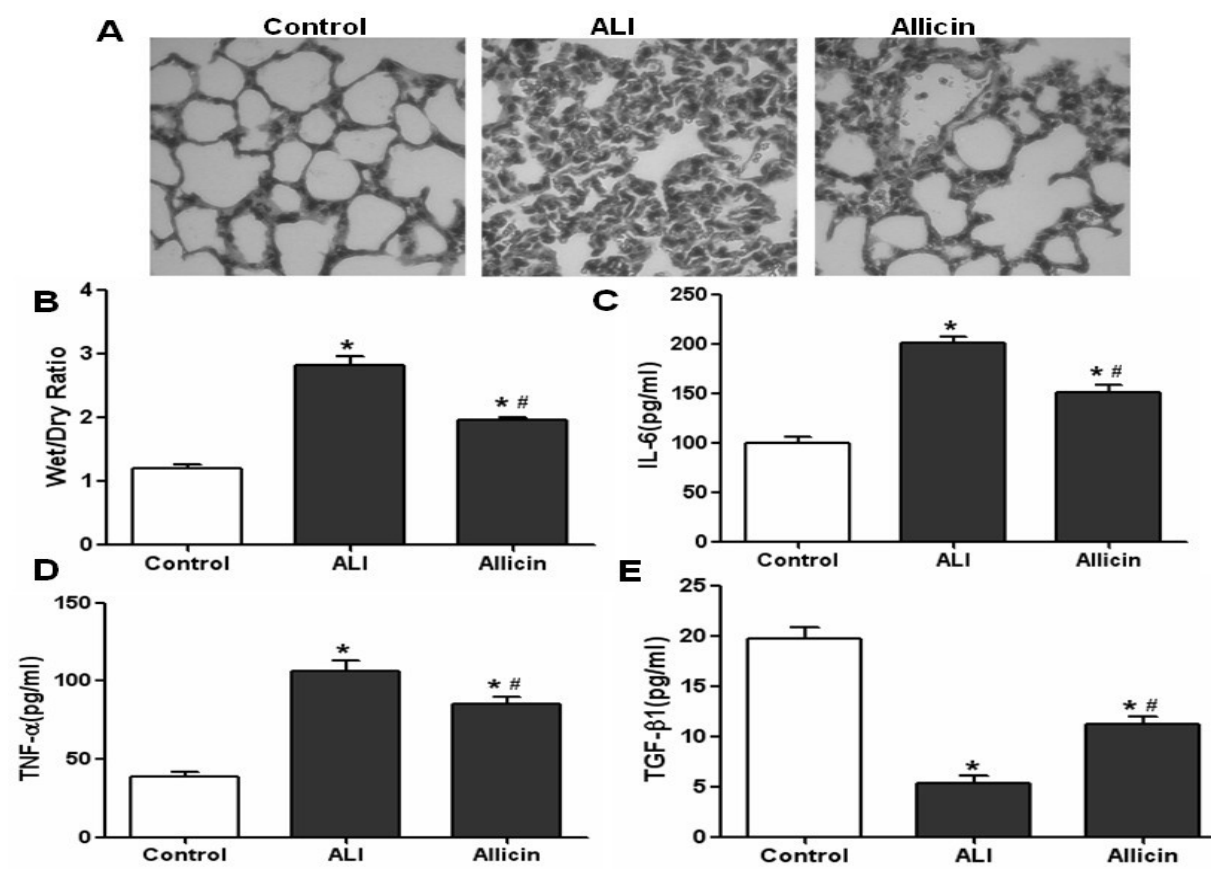

Fig 1: Allicin attenuated ALI in rats stimulated by LPS in vivo. (A) Lung tissue sections were stained with hematoxylin and eosin (H\&E) to determine the pathological changes with or without allicin treatment. The figures demonstrate representative views (x 200) from each group. (B) After LPS injection with or without allicin treatments, rats were sacrificed and their left lower lungs were obtained for wet/dry weight ratio determination; ${ }^{*} p$ $<0.05$ vs control; \# $p<0.05$ vs ALI. (C to E); ELISA was performed to determine the expression of TNF- $\alpha$, IL-6 and TGF- $\beta 1$ in BALF in the rats. ${ }^{*} p<0.05$ vs control; $\# p<0.05$ vs ALI
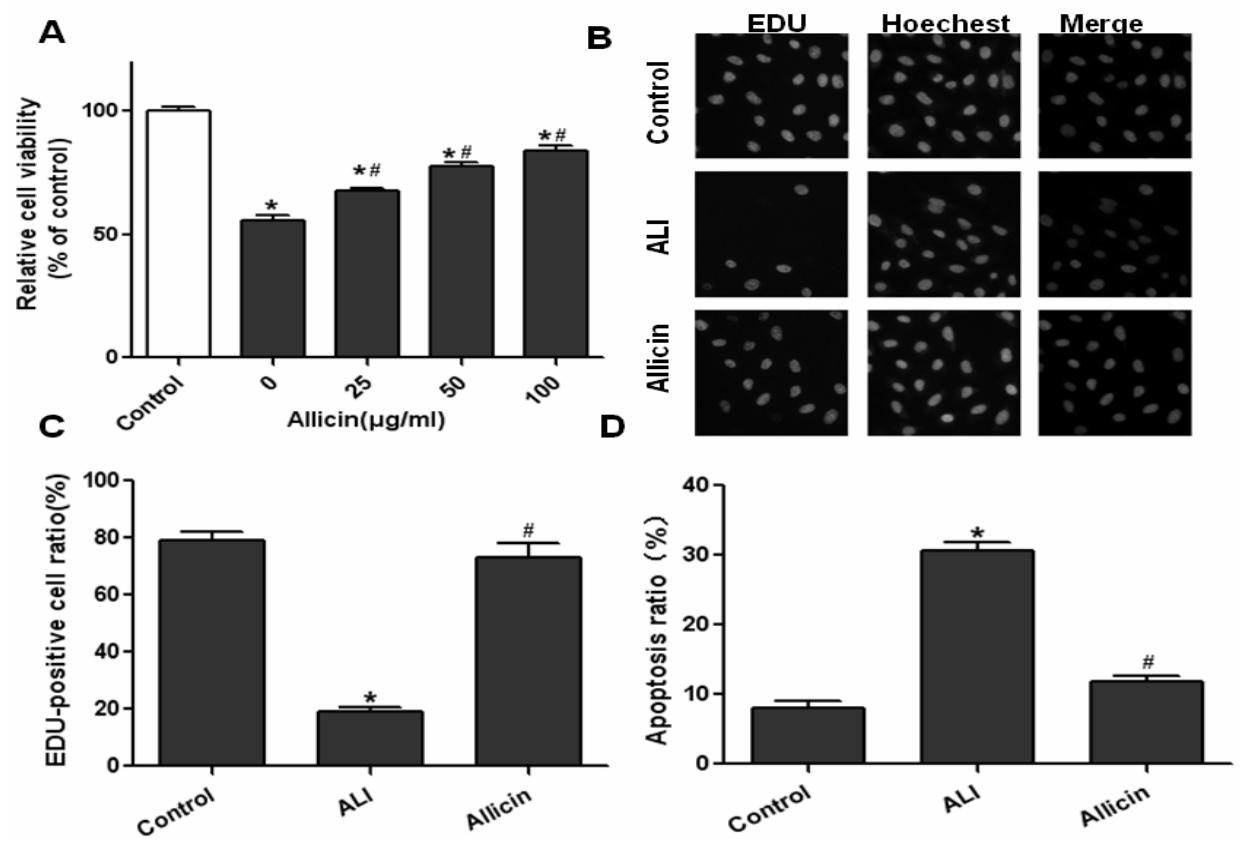

Fig 2: Effect of allicin administration on $A 549$ cell viability and apoptosis. (A-C) Cells were treated with allicin at different concentrations of allicin $(0 \mu \mathrm{g} / \mathrm{ml}, 25 \mu \mathrm{g} / \mathrm{ml}, 50 \mu \mathrm{g} / \mathrm{ml}$ and $100 \mu \mathrm{g} / \mathrm{ml})$ followedby CCK-8 and EdU incorporation assay to measure A549 viability; * $p<0.05$ vs control; \# $p<0.05$ vs ALI; (D) A549 cells were administered allicin and apoptosis rate was detected by flow cytometry; ${ }^{*} p<0.05$ vs control; \# $p<0.05$ vs ALI 
Furthermore, the in vitro study showed that claudin-4 was markedly reduced when A549 cells were incubated with LPS. However, incubating with allicin significantly increased claudin-4 expression in a dose dependent manner (Fig 4A and B). Taken together, these data demonstrate that allicin treatment resulted in a significant up-regulation of claudin-4 expression in vivo and in vitro.

\section{DISCUSSION}

LPS causes acute lung injury which is associated with the activation of macrophages, an increase in alveolar-capillary permeability and neutrophil influx into the lungs. Allicin is a biologically active component of garlic and has been shown to possess antimicrobial, antioxidant and immunoregulatory activities [9]. In the present study, we investigated the therapeutic effect of allicin in LPS-induced ALI and its underlying mechanism.

Pulmonary edema is a typical pathological changes in $\mathrm{ALI}$, leading to reduction of lung compliance and deterioration of pulmonary gas

A
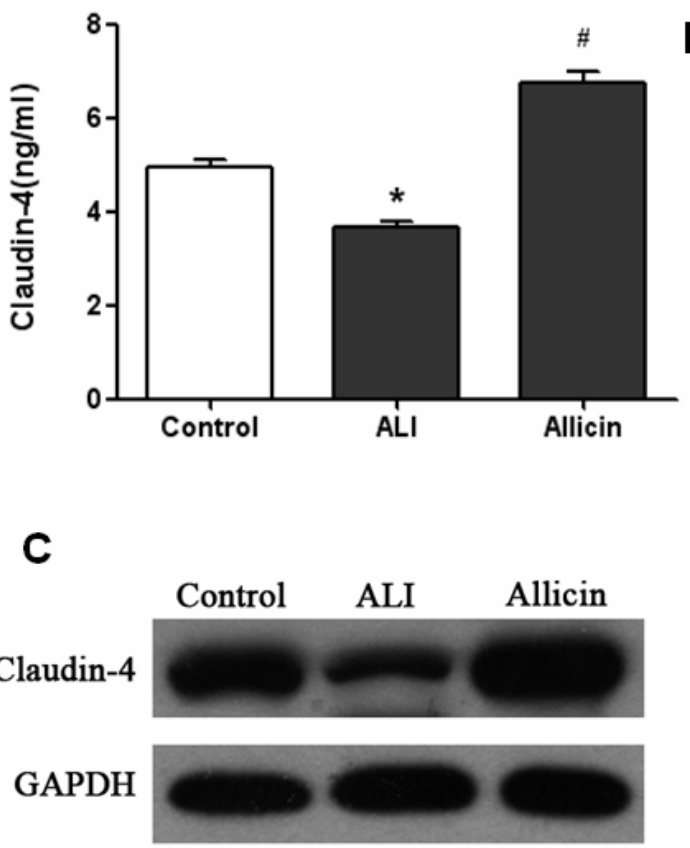

B

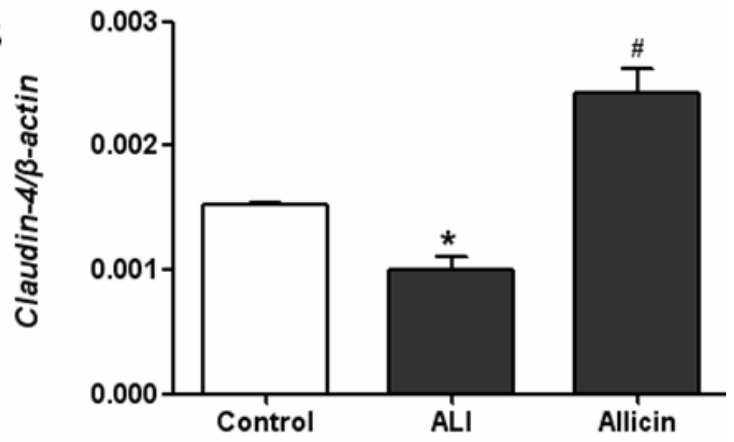

exchange [10]. Our study showed that the classical ALI pathological changes were reduced by allicin administration. In addition, we found that allicin significantly attenuated LPS-induced pulmonary edema in vivo as shown by lung wet/dry weight ratio. Numerous studies indicate that various inflammatory mediators were involved in the pathogenesis of ALI.

Among them, TNF- $\alpha$ and IL- 6 were considered as the most important inflammatory mediators in innate immune response [11]. Transforming growth factor $\beta$ (TGF- $\beta$ ) has been shown to play an anti-inflammatory role in immunity and autoimmunity [12].

According to our data, allicin treatment obviously reduced the up-regulated TNF- $\alpha$ and $\mathrm{IL}-6$ in BALF induced by LPS stimulation. Moreover, the anti-inflammatory mediator TGF- $\beta 1$ was decreased in BALF in the rats with LPS injection, which was reversed by allicin treatment. Taken together, these findings indicate that allicin effectively inhibited LPS-induced pulmonary edema and inflammation in vivo.

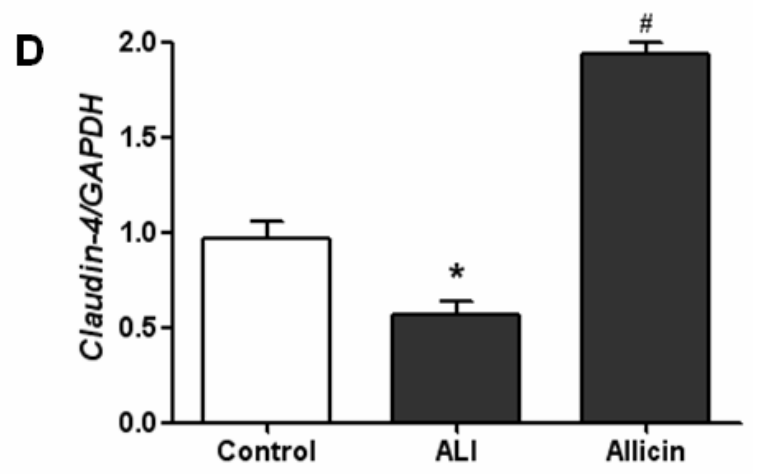

Fig 3: Allicin treatment increased expression of claudin-4 in BALF and lung tissues in rats; (A) After LPS injection with or without allicin treatments, cells in the BALF were collected and subjected to ELISA to measure the expression of claudin-4 in BALF in the rats; * $p<0.05$ vs control; \# $p<0.05$ vs ALI; (B) The mRNA expression of claudin-4 in the lung tissues was determined by real time PCR with $\beta$-actin used as a control; ${ }^{*} p<0.05$ vs control; \# $p<0.05$ vs ALI; (C-D) Claudin-4 expression in the lung tissues was analyzed by Western blot using anti-claudin-4 and anti-GAPDH antibodies. The expression of each protein was measured by relative band intensities; * $p<0.05$ vs control; \# $p<0.05$ vs ALI 

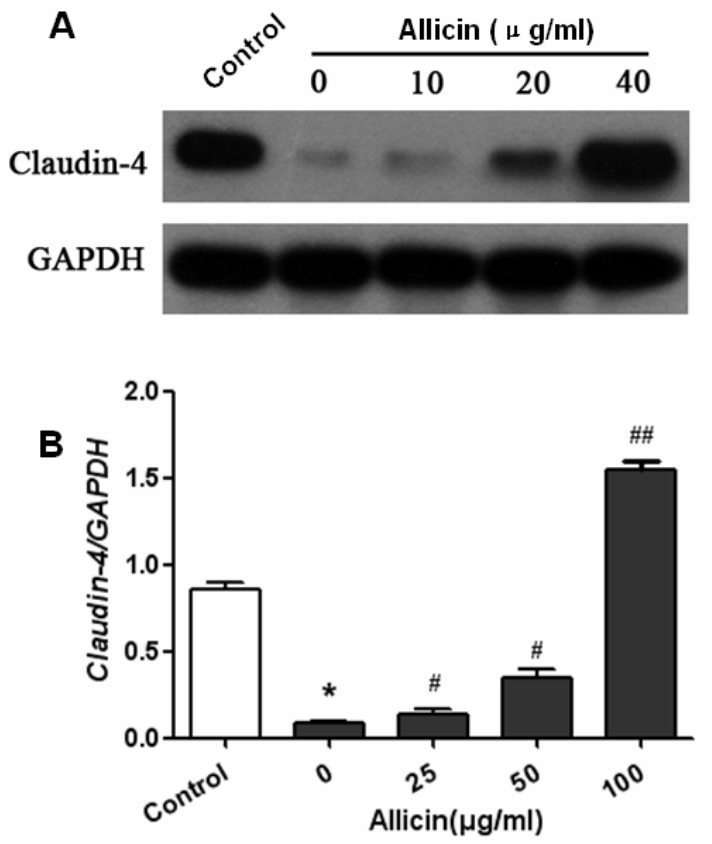

Fig 4: Allicin treatment promotes claudin-4 expression in vitro; cells were administrated with allicin at different concentrations. Thereafter, cells were lysed and protein was extracted for determination of claudin-4 (A and $B$ ) expression by Western blot using anti-claudin- 4 and anti-GAPDH antibodies. The expression of each protein was measured by relative band intensities; * $p$ $<0.05$ vs control; \# $p<0.05$ vs ALI

The in vitro effect of allicin was investigated using the lung epithelial cell line A549. Our data revealed that allicin significantly improved the A549 cell viabilities in a dose dependent manner as measured by CCK-8 and EdU assays. Apoptosis is the process of programmed cell death that plays a crucial role in cell growth and maintaining cellular homeostasis [13,14]. Allicin treatment significantly reduced the apoptosis rate of A549 cells as shown by flow cytometry. In vitro, our results demonstrated that allicin administration promoted cell viability and reduced cell apoptosis, thus protecting against LPS-induced lung injury.

The molecular mechanism underlying the protective effect of allicin on LPS-induced ALI was further investigated. Claudins are a family of proteins that are the most important components of the tight junctions, where they establish paracellular barrier controlling the flow of molecules between the cells of an epithelium [15]. Claudin-4, one member of the claudins family, was reported to be required for maximal epithelial barrier function, including alveolar fluid clearance in mice $[16,17]$. The levels of claudin-4 can influence paracellular transport by conferring relative chloride selectivity and decreasing ion conductance, suggesting that claudin-4 plays a role in determining alveolar fluid clearance rates in human lungs [18]. In our study, claudin-4 expression was significantly increased with allicin administration in vivo and in vitro, suggesting that the protective effect of allicin was partly mediated by claudin-4.

\section{CONCLUSION}

The present study, for the first time, demonstrates that allicin can protect against LPS-induced $A L I$ in vivo and in vitro probably by up-regulation of claudin-4 expression.

\section{ACKNOWLEDGEMENT}

This study was supported from public welfare funds of Science and Technology Department of Zhejiang Province (2013C33G1360121), general project funds of Health Department of Zhejiang Province (2012Kyb012) and general project funds of Administration of Traditional Chinese Medicine of Zhejiang Province (2012ZA018).

\section{REFERENCES}

1. Sung Kyoung Kim, Chan Kwon Park, Sung Hak Park, Young Kyoon Kim. Effects of Rosiglitazone on the Expression of PPAR-y and on the Production of IL-6 and IL-8 in Acute Lung Injury Model Using Human Pulmonary Epithelial Cells. Trop J Pharm Res 2011; 10 (6): 731-738.

2. Miyoshi K, Yanagi S, Kawahara K, Nishio M, Tsubouchi $H$, Imazu Y, Koshida R, Matsumoto N, Taguchi A, Yamashita S. Epithelial Pten controls acute lung injury and fibrosis by regulating alveolar epithelial cell integrity. Am J Respir Crit Care Med 2013; 187: 262275.

3. Kolliputi N, Waxman AB. IL-6 cytoprotection in hyperoxic acute lung injury occurs via suppressor of cytokine signaling-1-induced apoptosis signal-regulating kinase-1 degradation. Am J Respir Cell Mol Biol 2009; 40: 314-324.

4. Mutlu GM, Budinger GR. Incidence and outcomes of acute lung injury. N Engl J Med 2006; 354: 416-417.

5. Antony ML, Singh SV: Molecular mechanisms and targets of cancer chemoprevention by garlic-derived bioactive compound diallyl trisulfide. Indian J Exp Biol 2011; 49: 805- 816..

6. Kim YS, Kim KS, Han I, Kim MH, Jung M H, and Park HK. Quantitative and qualitative analysis of the antifungal activity of allicin alone and in combination with antifungal drugs. PLoS One 2012; 7: e38242. 
7. Zhang $X$, Liu F, Liu H. Urinary trypsin inhibitor attenuates lipopolysaccharide-induced acute lung injury by blocking the activation of p38 mitogen-activated protein kinase. Inflamm Res 2011; 60: 569-575.

8. Derrell C. Guide for the care and use of laboratory animals. Institute of Laboratory Animal Resources. National Academy Press, Washington DC, USA 1996.

9. Chan JY, Yuen AC, Chan RY, and Chan SW. A review of the cardiovascular benefits and antioxidant properties of allicin. Phytother Res 2013; 27: 637-646.

10. Suresh R, Kupfer $Y$, Tessler S. Acute respiratory distress syndrome. N Engl J Med 2000; 343: 660-661.

11. Jayasooriya RGPT, Chang-Hee Kang, Yung Hyun Choi, Woo Shin Ko. Aqueous Extract of Oldenlandia diffusa Suppresses LPS-Induced iNOS, COX-2 and TNF- $\alpha$ Expression in RAW 264.7 Cells via the NF-KB Activity. Trop J Pharm Res 2011; 10 (4): 403-411

12. Sanjabi S, Zenewicz LA, Kamanaka $M$, and Flavell $R A$. Anti-inflammatory and pro-inflammatory roles of TGFbeta, IL-10, and IL-22 in immunity and autoimmunity. Curr Opin Pharmacol 2009; 9: 447-453.
13. Shaham S. Apoptosis: a process with a (beta) NAC for complexity. Cell 2003; 114: 659-661.

14. Inoue S, MacFarlane M, Harper N. Histone deacetylase inhibitors potentiate TNF-related apoptosis-inducing ligand (TRAIL)-induced apoptosis in lymphoid malignancies. Cell Death Differ 2004; 11 Suppl 2: S193-S206.

15. Wray C, Mao Y, Pan J. Claudin-4 augments alveolar epithelial barrier function and is induced in acute lung injury. Am J Physiol Lung Cell Mol Physiol 2009; 297: L219-L227.

16. Lipschutz JH, Li S, Arisco A, Balkovetz DF. Extracellular signal-regulated kinases $1 / 2$ control claudin-2 expression in Madin-Darby canine kidney strain I and Il cells. J Biol Chem 2005; 280: 3780-3788.

17. Van Itallie C, Rahner C, Anderson JM. Regulated expression of claudin-4 decreases paracellular conductance through a selective decrease in sodium permeability. J Clin Invest 2001; 107: 1319-1327.

18. Rokkam D, Lafemina MJ, Lee JW. Claudin-4 levels are associated with intact alveolar fluid clearance in human lungs. Am J Pathol 2011; 179: 1081-1087. 